\title{
Gray Box Time Variant Clogging behaviour and Pressure Drop Prediction of the Air Filter in the HVAC System
}

\author{
Hossein Alimohammadi ${ }^{1}$, Kristina Vassiljeva ${ }^{1}$, Eduard Petlenkov ${ }^{1 *}$, Martin Thalfeldt ${ }^{2}$, Alo Mikola ${ }^{2}$, Tuule Mall Kull ${ }^{2}$, \\ and Ahmet $\mathrm{Köse}^{1,3}$ \\ ${ }^{1}$ Centre for Intelligent Systems, Department of Computer Systems, Tallinn University of Technology, 19086 Tallinn, Estonia \\ ${ }^{2}$ Nearly Zero Energy Buildings Research Group, Department of Civil Engineering and Architecture, Tallinn University of \\ Technology, 19086 Tallinn, Estonia \\ ${ }^{3} \mathrm{R} 8$ Technologies company, Tallinn 11415, Estonia
}

\begin{abstract}
Identification and prediction of clogging behavior in heating, ventilation, and air conditioning (HVAC) filters is crucial to avoid issues such as system overheating, energy waste, lower indoor air quality, etc. Researchers are focusing more on the particle loading characteristics of a filter medium in a laboratory environment under steady-state conditions, fixed particle concentrations, area of porosity, dust feed and volumetric flow rate. However, recent research still shows uncertainties in modeling as well as the implementation problems of constructing the HVAC laboratory test bench and equipment. In addition, subjects such as non-uniform particle deposition depreciation of the condition and various type of mechanical filters such as fibrous, fabric, granular, and membrane filter or electrostatic filters which typically used in HVAC systems perform under some assumptions and still need more research. The studies become even more difficult acquiring a large number of time-varying and noisy signals. Another approach among studies is data-driven knowing that Building Automation System (BAS) is not equipped with appropriate sensor measuring the clogging, it is needed to drive the clogging mathematical model from the pressure drop signal. This paper bridges the gap between particle-size study and black box modeling of HVAC filter which has not received much attention from authors. The proposed method assumes that the pressure drop is the result of two time-varying functions; $f(t)$, which represents the dynamics of clogging and, $g(t)$, which refers to dynamics of remained terms. The exponential and polynomial of second order functions are proposed to express the clogging behavior. The software package based on Particle Swarm Optimization Artificial Bee Colony (PSOABC) algorithm, is developed and implemented to estimate the coefficients of the clogging functions based on smallest RMSE, high coefficient of correlation and acceptable tracking. Five Air Handling Unit (AHUs) are selected for practical verification of the model and the results show that the applied method can successfully predict clogging and pressure drop behaviour of HVAC filters.
\end{abstract}

\section{Introduction}

HVAC systems are usually installed in public and private buildings. A clogged HVAC filter can severely damage the HVAC system, leading to high costs. Since the system is closed loop, the airflow will be minimized and the blower will work harder to pass the right amount of air through the coil or heat exchanger when the filter is clogged. Although some HVAC systems have reusable filters that can be cleaned, dried, and reinserted, the disposable filters used in many systems must be replaced regularly. Filter clogging is caused by changes in filter geometry over time. In other words, the size of the porosity in the filter is changing. The identification and prediction of the clogging behavior of the HVAC filter is essential to avoid problems such as system overheating, energy waste, indoor air quality reduction, system efficiency reduction, and short cycle of thermostat based system, freezing, fuel and repair costs, etc. Researchers pay more attention to the particle loading characteristics of filter media in laboratory environments. The articles are usually performed under steady-state conditions, that is, under a fixed particle concentration, different type of porosity, dust feed amount and volumetric flow rate, and a particle size model which is more useful to the designer will be obtained throughout the process.

However, the uncertainty in modeling and the implementation of HVAC laboratory test benches and equipment, as well as the issue of non-uniform particle deposition covering the HVAC filter plate, are not very useful for overhaul and maintenance purposes. Another disadvantage is that it is usually impossible to have a perfect model since operating conditions, depreciation of conditions and various components can cause changes in the system. Especially when the results of research should be used in the field where the overhaul period needs a fast, reliable, and time to change or predict the clogged filter. In addition, the success of translating data collected in a laboratory environment to

\footnotetext{
*Corresponding author: eduard.petlenkov@taltech.ee
} 
predict filter media performance has been very limited. In addition, there are many time-varying and noisy signals from BIM or BAS, which makes the processing process very time-consuming and slow. Furthermore, all components are located in a closed-loop HVAC system are coupled. Changes in one component will affect other components, which may affect the independence of literature research methods.

Another approach among researches concern to clogging is data-driven. Since there is no dedicated clogging sensor, additional processing is required to separate and extract the clogging mathematical model from the pressure drop mathematical model. This is because HVAC is only equipped with a differential pressure sensor and only uses BAS to receive pressure data. In other words, black-box modeling is not appropriate, since the data obtained by the BAS system is a pressure drop signal. The output of the black box will be a pressure drop and is not reliable enough to extract the clogging behavior.

This paper bridges the gap between particle-size study and black box modeling of HVAC filter. The estimation of parameters of function expresses the dynamics of HVAC clogging filter has not received much attention from authors and it is necessary to investigate the performance of clogged filter under the real-world conditions and noisy signals in a quick and reliable method. One of the advantages of this method is that the data acquired from BIM can be processed directly and can be implemented and run in the field for prediction. It covers unsteady state behavior, uneven particle deposition, and other uncertainties, which are usually eliminated under particle-size assumptions. It is assumed in the proposed method the pressure drop is the result of two time-varying functions; $\mathrm{f}(\mathrm{t})$, which represent the dynamics of clogging and, $g(t)$, which refers to dynamics of remained terms such as volumetric flow velocity, pressure dynamics, and temperature dynamics. An exponential pattern and a polynomial specifically a quadratic function are proposed to express the clogging behavior. The software package based on Particle Swarm Optimization Artificial Bee Colony (PSOABC) optimization algorithm is developed and implemented to estimate the coefficients of the clogging functions based on less RMSE, high coefficient of correlation and acceptable tracking.

The structure of the article is as follows: In Section 2, the background of clogging in the HVAC filter is presented and it is classified into the mathematical particle size approach of pressure drop in the clean and clogged filter as well as data-driven approach. Section 3 presents a different approach to the filter model and parameter estimation. The reasons for choosing this method are discussed and the prediction result is presented using different data from other AHUs. In Section 5, the conclusion of the article is shown.

\section{Background}

Many studies have shown that the filter load or clogging behavior (for example, the pressure drop and the particle mass/volume area per unit area of the filter) is affected by the properties of the particles (for example, particle size, distribution, and particle concentration), filter media (such as fiber, fabric, granular and membrane filter) and other environmental conditions (such as relative humidity, temperature).

For a High Efficiency Particulate Air (HEPA), surface filtration is dominant. The loaded particles usually form a dust cake (Fig. 1). The total pressure drop across the clogged filter consists of two parts. One is caused by the pressure drop in the cleaning filter $(\triangle P O)$, and the other is caused by the dust filter cake $(\Delta P C)$. Therefore, the pressure drop when the filter is clogged can be written as

$$
\Delta P=\Delta P_{0}+\Delta P_{c}
$$

Which $\Delta P_{0}$ is pressure drop of the clean filters or initial pressure drop and $\Delta P_{c}$ refers to pressure drop into clogged filters.

\subsection{Pressure drop in clean filters}

Many relationships have been proposed to calculate the pressure drop across clean fiber filters. A fundamental model is based on the sum of all fiber resistances. At low Reynolds number, the drag force on the cylindrical fiber array can be approximated as the product of drag coefficient, viscosity, and flow velocity. Werner and Clarenburg [1] conducted extensive experiments to test glass fiber filters, but did not consider the random orientation of the fiber cylinders and the flow interference between them. It must be mentioned that these equations are semi-empirical and do not consider changes in fiber size. The study of Jackson and James [2] also showed that when the packing density is low, the prediction begins to deviate from the experimental test. Hosseini and Tafreshi [3] developed a virtual 3-D model to simulate the internal microstructure of the fiber structure to predict and simulate the pressure drop and collection efficiency of the cleaning filter. However, in addition to being challenging precision mesh, simulation software is expensive.

\subsection{Pressure drop in clogged filters}

The pressure drop on the clogged filter has been extensively studied. Some researchers have tried to establish mathematical models to simulate a pressure drop of the clogged filter by solid particles under different filtration modes. At the microscopic level, the increase of pressure drop across a clogged filter is due to the additional flow resistance caused by the deposited particles. Passing through the research of other authors, Hiragi and Kanaoka [4] proposed a theoretical model based on drag theory to predict the pressure drop of dustclogging the filter. They found that the model is only applicable if the given effective fiber diameter and the drag coefficient of the clogged fiber are a function of the cumulative amount of captured particles. Another method developed by Bergman et al. to collect particles through filter media[5], who assumed that in the particle deposition process, the filter may be regarded as a medium with two types of fibers. 
However, compared with experimental data, Vendel et al. [6] showed that the pressure drop is underestimated during the clogging process of particle in the Bergman model. They suspect that the reason may be due to the assumption that the particles in the filter media are deposited uniformly and have uniform diameters. Experimentally and theoretically, a lot of work has been done to study the pressure drop uniformly on the dusk cake piled on the surface of the filter. Endo and Chen [7] proposed (2) to calculate the pressure drop of the dust cake in the case of dust cake in consideration of the polydispersity and shape of the particles:

$$
\Delta P_{\{c\}}=18 \mu u_{s} H \frac{(1-\varepsilon) v(\varepsilon)}{\varepsilon^{2}} \frac{k}{d_{v g}^{2} \exp \left(4 \ln ^{2} \sigma_{g}\right)}
$$

where $\mu, u_{s}, H, \kappa, d_{v g}$ and $\sigma_{g}$ are fluid viscosity, superficial velocity, cake height, dynamic shape factor of particles, and the geometrical mean diameter and standard deviation of test particles, respectively. $\varepsilon$ and $v(\varepsilon)$ are the cake porosity and void function.

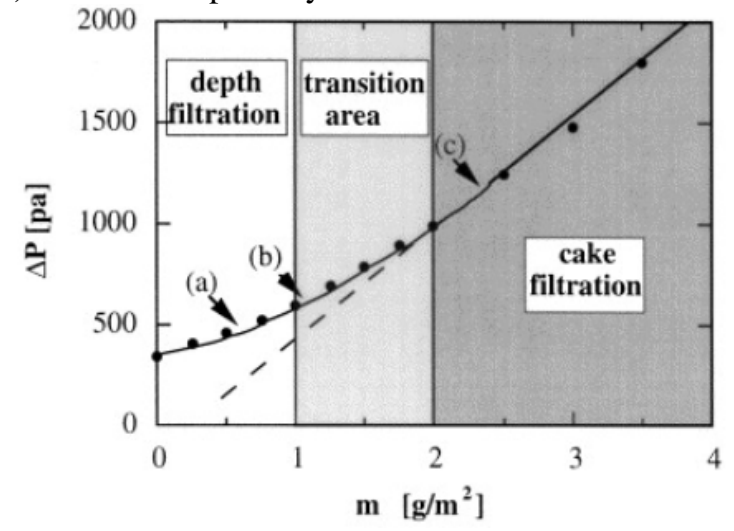

Fig. 1. Behavior of increase in pressure drop vs. particle per unit filtration area during clogging. [8] Copyright 1999, Elsevier.

Particle deposits depends on the test filter, and their evolution was observed using scanning electron micrographs, [8]. The increase in pressure drop can be described in terms of two main steps (Fig. 1). In the first stage, the development is slow, and in the second stage, the growth is quite rapid and linear.

\subsection{Mathematical modeling of filter in HVAC}

In order to drive the mathematical model of the HVAC filter, it should be assumed that the mass flow rate through the inlet must be exactly the same as the mass flow rate through the outlet. It is acceptable to assume that heat exchange does not occur between the wall and the environment. In addition, no work is done on or by fluid. The energy flow through inlet must be exactly equal to the energy flow through outlet. External forces on the fluid include those due to pressure and those caused by viscous friction in component walls. Body forces like gravity are ignored. The expression of friction forces in terms of a loss factor $\xi$ yields the semiempirical expression. This type of formulation is to allow for a change in sign as the direction of the stream:

$$
\Delta p=\xi \frac{\dot{m}|\dot{m}|}{2 \rho S^{2}}
$$

where:
$\Delta p$ is the pressure drop through input and output of filter, $\xi$ is the loss factor. Fluid density describes by $\rho$ and the flow area by $S$.

It is assumed that the resistance is adiabatic. It does not exchange heat with the environment. The pressure drop is proportional to the square of the mixture mass flow rate (dry air, water vapor and other gas like CO2) and inversely proportional to the mixture density.

Applied ideal gas law, $\rho=\frac{p_{\text {inlet }}}{R T_{\text {inlet }}}$, the equation (3) can be rewritten as follows:

$$
\Delta p=\frac{\xi}{2 S^{2}}\left(\dot{m} \sqrt{\dot{m}^{2}+\dot{m}_{\text {lam }}^{2}}\right) \frac{R T_{\text {inlet }}}{p_{\text {inlet }}}
$$

$\dot{\mathrm{m}}_{\text {lam }}$ mixture mass flow rate threshold for laminar flow and is equal to

where:

$$
\dot{m}_{\text {lam }}=f_{\text {lam }} \times \dot{m}_{*}
$$

$f_{\text {lam }}$ - fraction of nominal mixture mass flow rate for laminar flow transition;

$\dot{\mathrm{m}}_{*}$ - nominal flow rate obtained from filter data sheet or empirical data;

$\mathrm{p}_{\text {inlet }}$ - inlet pressure $\mathrm{Pa}$;

$\mathrm{T}_{\text {inlet }}$ - inlet temperature $\mathrm{K}$;

$\rho$-inlet temperature $\mathrm{kg} / \mathrm{m}^{3}$;

$\mathrm{R}_{\mathrm{a}}$ - dry air specific gas constant, 287.048; J/(kgK);

$\mathrm{R}_{\mathrm{w}}$ - water specific gas constant, 461.52; J/( $\left.\mathrm{kgK}\right)$;

$\mathrm{R}_{\mathrm{g}}$ - trace gas specific gas constant $\left(\mathrm{CO}_{2}\right), 188.92 ; \mathrm{J} /$ (kgK);

The $R=\left(R_{a}+R_{w}+R_{g}\right) / 3$ is mixture specific gas constant.

\subsection{Data driven approach}

In another type of modeling approach for clogged filter, the system can be considered as a black box, and measurements can be made on the input and output of the system. However, due to the limitation of the sensor, only the pressure drop of the filter can be modeled, and the filter clogging cannot be modeled. Linear and nonlinear mathematical equations can be adapted to the measured data, resulting in a data-driven model. Physicbased models have better generalization capabilities, while data-driven models have higher accuracy on training data. Despite the fact that the black box model has high accuracy, but it is not necessary to understand the physics of the system, which leads to low generalization ability. The white box or particle size model, has good prediction accuracy under various operating conditions, but since no measurement method is used in the development process, its accuracy is low. Gray-box models use both measured values and system physical characteristics at the same time, so they provide good accuracy and high generalization ability, but the development of gray-box models requires more work. The gray box models have been designed and developed for air handling units (AHU) [9], zones [10], vapor compression cycles [11], chillers [12], heat load estimation [13], [14], heat exchangers [15], and heat pumps [16].

Genetic algorithm (GA) is a kind of the metaheuristic algorithm that has been increasingly used in various 
HVAC problems, such as control component optimization [17], system design optimization [18], chiller optimization [19], and air side optimization [20]. Among metaheuristic optimization, artificial bee colony (ABC) has drawn a lot of attention in recent years. The $\mathrm{ABC}$ algorithm attempts to optimize the problem by imitating the foraging behaviour of bees, and has begun to be applied to engineering optimization problems. However, there are not many articles about ABC in the HVAC application. Zang et al, [21] focus on the newly developed meta-algorithm based on the ABC paradigm and its effectiveness and efficiency on HVAC problems. They evaluated the effectiveness and efficiency of both artificial bee colony algorithm with one-position inheritance (OPIABC) and $\mathrm{ABC}$ against that of GA through heat exchanger.

\section{New approach to filter clogging and pressure drop prediction}

As it is mentioned earlier, due to the limitation of the sensor, only the pressure drop of the filter can be modeled as black-box, not the clogging of the filter. In addition, while a lot of work has been done in the modeling and verification of HVAC systems, it is difficult to find a universal model that is useful for all different types of systems in infinitely possible configurations. In order to be able to simulate and modify the system correctly, it is necessary to write the static and dynamic equations of each subsystem and perform measurements to identify the parameters.

\subsection{Infinite problem}

In equation (4), the first terms represent the clogging function. The term $\mathrm{S}$ changes over time since the filter porosity area changes during clogging. The term $\xi$, represent the drag force at low Reynolds number which also changes during time, Werner and Clarenburg, [1].

Considering gas law, and density equation and by rewriting equation (4), and assuming equation (6), the clogging can be expressed by dynamic functions related to pressure drop, input pressure and temperature, and volumetric flow rate.

$$
\begin{gathered}
\frac{\xi}{2 S^{2}}=f(t)_{\text {clog }} \\
f(t)_{\text {clog }}=\frac{\Delta p}{\dot{v} \sqrt{\dot{v}^{2}+\dot{v}_{\text {lam }}^{2}} \frac{p_{\text {inlet }}}{R T_{\text {inlet }}}}
\end{gathered}
$$

Where $\dot{v}$ is flow rate, $\mathrm{m}^{3} / \mathrm{s}$. Since $\dot{v}$ is located in denominator, the output in the case of no flow rate can be diverge. It is obvious that $f(t)_{\text {clog }}$ is clog changes due to molecular aggregation over a period of time, and may lead to product quality degradation and processing time extension, resulting in energy loss.

According to (7), measured pressure, temperature, and flow data are required to obtain the clogging behavior. Fig. 2. shows the data of one of the studied AHUs acquired from BAS unit. One approach is to directly divide data and extract clogged behavior. The problem with this approach is that the data is noisy, mispointing and sometimes contains step time lead or lag (which is equal to 15 minutes in the studied AHU) and the result of calculation based on (7) leads to an infinite result in some sample points and will prolong the prediction process. In addition, using an electronic highpass filter (HPF) or notch filter with a specific bandwidth to eliminate these noises can deform and shift the main signal and reduce the accuracy of the results.

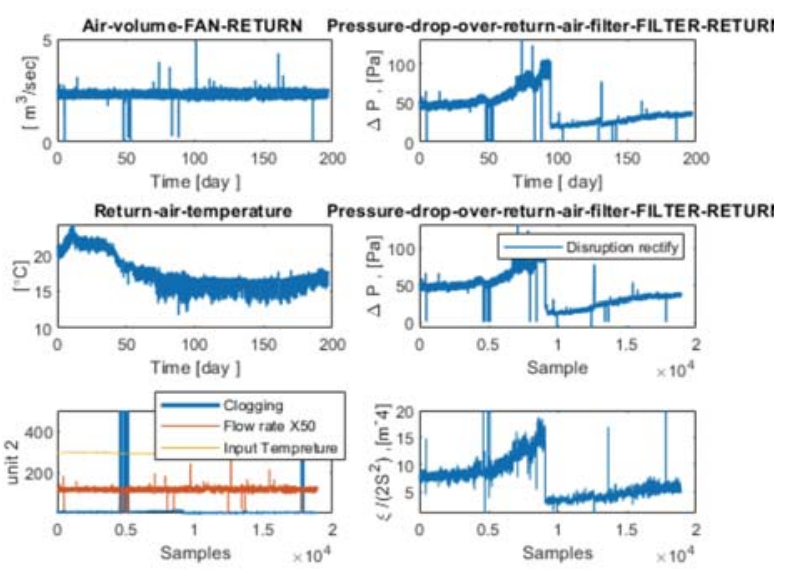

Fig. 2. Not applicable clogging formula in real data

Fig. 2. also shows the sudden pressure drop which is due to the replacement of the filter. However, it has been seen that the pressure drop behaviour has decreased, which can be considered as a uniform distribution of clogging in the panel. Also slight vibrations or shake of the filter can cause this drop. If the slope of the graph after the filter replacement is higher than before, it can be due to the normal distribution of dust by the occupants. There are other unusual behaviours in the measured data that are not mentioned in this article.

In this article, we have developed a method for modeling and estimating filter parameters, which can usually be extended to other HVAC subsystems and integrated to simulate larger systems. The developed model is suitable for the new controller design on local and supervisory level.

Reasons for choosing this method are:

- The articles are usually accomplished under steady-state conditions, and the whole process is analyzed with a fixed particle concentration, area of porosity, dust feed and volumetric flow rate throughout the process. Various types of mechanical filters such as fibrous, fabric, granular, and membrane filter or electrostatic filters which typically used in HVAC systems perform under some assumptions and still need more research.

- The studies become even more difficult acquiring a large number of time-varying and noisy signals.

- Particle testing methods are very limited for specific filters and sometimes require expensive equipment

- It is impossible to perform a micro and particle-size inspection on site to find the pressure drop

- The amount of data received from BAS is 
very large, which takes a lot of time to predict.

- Unable to measure the particle mass concentration or appropriate detector or sensor which directly monitor the clogging behavior

As it is mentioned in the previous section, noisy data and missing point signals cause the clogging function to not be extracted properly, and no similar paper was found on the estimation of coefficients of HVAC filters. Therefore, the new approach presented in this article is based on expressing clogged behavior as a mathematical equation and finding its coefficients by parameter estimation methods.

In order to avoid the divergence of the denominator in (7), polynomial and exponential functions have been suggested, and then the coefficients of these functions have been obtained through intelligent methods.

$$
\begin{gathered}
f(t)_{\operatorname{clog} 1}=K_{1} t^{2}+K_{2} t+K_{3} \\
f(t)_{\operatorname{clog} 2}=L_{1} e^{L_{2} t}+L_{3}
\end{gathered}
$$

It should be noted that the terms $\mathrm{K}_{1,2}$ or $\mathrm{L}_{1,2}$ in (8) and (9) express the pressure drop of clogged filter, and the last terms $\mathrm{K}_{3}$ and $\mathrm{L}_{3}$ express the pressure drop of clean filter.

The input of the model is measurement data, including volume flow rate, inlet temperature and inlet pressure, and the output is measured pressure drop. The parameter estimation process is formulated as a nonlinear optimization problem. For this purpose, an objective function (fitness function) is used to minimize the error between simulation and empirical data. The GA, PSO, ABC, PSOGA and PSOABC algorithms were used to estimate the parameters.

\subsection{PSOABC approach for parameter estimation}

The next step is to build a machine learning platform to estimate model parameters using experimental data. The idea was to develop a software package. The package includes a combination of scripts and graphical models.

The advantage of the graphical environment is:

- It can make modeling so fast, easy to understand and debug

- $\quad$ Enable the use of Simscape environment, a very powerful library in MATLAB

The package estimates unknown parameters in SISO and MIMO models. It is also possible to apply to system identification purposes e.g. control optimization.

Mfile scripts are used to implement the algorithms. In order to communicate between the mfile and the graphical model, some suitable code are used. It is also capable to estimate several unknown parameters simultaneously. There are many algorithms to test. The results showed that $\mathrm{ABC}$ and $\mathrm{PSOABC}$ require less iteration to achieve acceptable tracking with high correlation coefficient and smallest RMSE. The selected $\mathrm{ABC}$ or PSOABC depends on the different AHU filters.
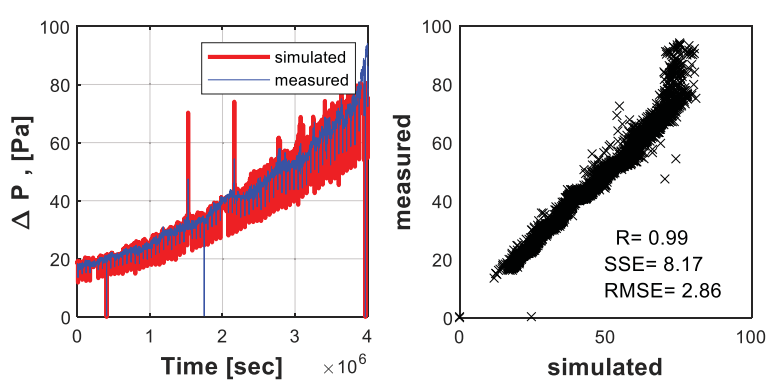

Fig. 3. Polynomial model result

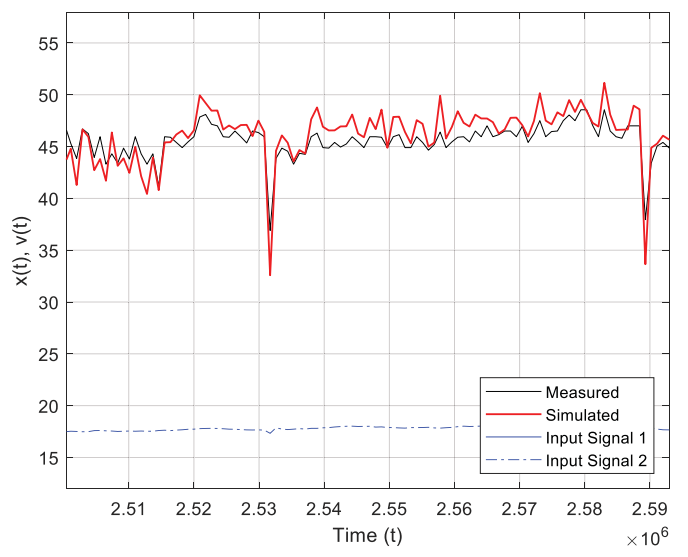

Fig. 4. Tracking of final result

Apart from the parameter estimation approach, it is necessary to check the graphs to make sure that the simulated data correctly trace the measured data (Fig. 4.). The reason is that the cost function is calculated by the RMSE equation. It has sometimes been observed that RMSE is low even with inadequate tracking. In other words, it is possible to have better RMSE with weak tracking or vice versa.

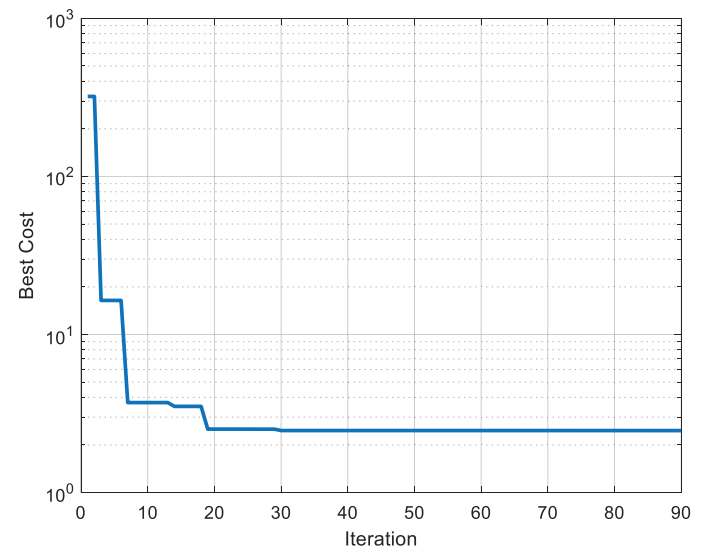

Fig. 5. Cost function history, clogging $(\xi)$ estimation

The plot in Fig. 5. relates to the process of minimizing the cost function, known as the evolution or convergence graph. The summary of comparisons among algorithm are listed in Table 1. The results of algorithms with different population numbers are summarized. In order to find the best solution, the root mean square error (RMSE) is calculated, which indicates the minimum error or minimum pressure difference between the simulated data and the measured data. The Pearson R correlation coefficient between the simulated data and the measured data is also calculated to observe the best tracking in addition to minimizing 
the error. Tracking is acceptable, which means that the unknown parameters obtained are reliable. The return filter was chosen since the clogging rate of the return filter is higher than the supply filter due to sources such as cooking, odorous materials, and air molecules with more surface groups. The comparison result shows that the PSOABC method with a population of 50 is the best solution. The PSOABC algorithm has been used in the next part of this research to estimate the parameters of filter clogging.

Table 1. Algorithm Comparison results.

\begin{tabular}{cccccc}
\hline Algorithm & GA & PSO & ABC & $\begin{array}{c}\text { PSO- } \\
\text { GA }\end{array}$ & $\begin{array}{c}\text { PSO- } \\
\text { ABC }\end{array}$ \\
\hline R & 0.95 & 0.94 & 0.95 & 0.95 & 0.99 \\
RMSE & 3.18 & 5.37 & 2.89 & 3.22 & 2.77 \\
\hline Pop size & 80 & 80 & 50 & 50 & 50 \\
\hline$f_{\text {lam }}$ & $800 \mathrm{e}$ & $761 \mathrm{e}-3$ & $400 \mathrm{e}-3$ & $687 \mathrm{e}-3$ & $420 \mathrm{e}-3$ \\
& -3 & & & & \\
\hline
\end{tabular}

The algorithm minimizes the cost function to estimate the clogging coefficient which is error of simulated and measured data. Table 2 shows the result of estimation of parameters of equations (8)-(9). The estimated clogged coefficients are as:

Table 2. Clogging estimation for different AHUs

\begin{tabular}{lll}
\hline \hline Unit & Polynomial & Exponential \\
\hline 2 & K1 $=1.2089 \mathrm{e}-13$ & L1 $=0.20098$ \\
& K2 $=0$ & L2 $=0$ \\
& K3 $=6.7344$ & L3 $=7.0652$ \\
& R $=0.94$ & R $=0.95$ \\
& RMSE $=5.38$ & RMSE $=4.44$ \\
5 & K1 $=3.37 \mathrm{e}-13$ & L1 $=0.17109$ \\
& K2 $=7.65 \mathrm{e}-07$ & L2 $=4.2036 \mathrm{e}-07$ \\
& K3 $=2.54$ & L3 $=6.1421$ \\
& R $=0.99$ & R $=0.98$ \\
& RMSE $=2.86$ & RMSE $=2.91$
\end{tabular}

\subsection{Measurement}

The measurement signals were obtained from a building information model (BIM) and building automation system (BAS) located in Tallinn (Fig. 6.). There are two filters, one in the supply air duct and the other in the return. Pressure drop measurement intervals are recorded every 15 minutes and the data are raw without any pre-signal processing. For this method, the parameter search region (upper and lower range of parameters) must be defined. To define the upper and lower limits, a region can be created using the parameter obtained from the manufacture datasheet under standard test conditions. However, in the case of an inaccessible datasheet, the expert can set the upper and lower limits in the range of the common value. Selecting an infinite range increases the estimated time and leads to other local optima.

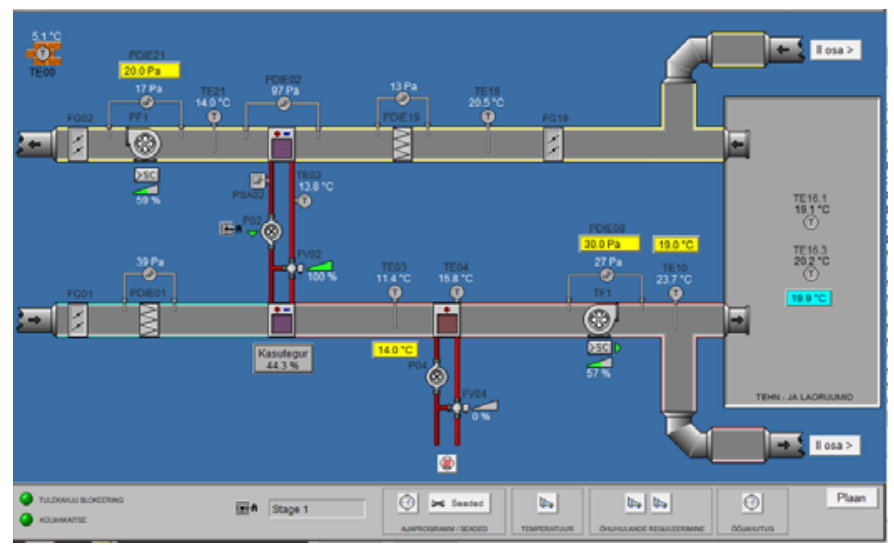

Fig. 6. Diagram of filter in AHU of HVAC system

\subsection{Prediction}

The next step is the prediction of pressure drop due to filter clogging. As the airflow of the AHU decreases when the filter is clogged, less and less air passes through the duct. The technician replaces the filter every year or twice according to the overhaul schedule. In many cases, during overhaul date, the filter is clean or very dirty, so all these cases will incur additional costs. Therefore, the prediction of pressure drop is very important in order to optimize the overhaul period and energy consumption as well as air quality in the HVAC system. The derived model is used to predict clogging and pressure drop behavior. Five AHUs is selected and the software has considered the 67 days for prediction. The results show that the applied method can successfully predict the HVAC filters. To prove this method, the additional experimental data have been used indicated that there is acceptable consistency between model and experimental data. The result shows that the polynomial pattern is more realistic for estimating the clogged filter. In the exponential pattern, the pressure drop increases dramatically, which is not very close to the actual site data. Sometimes a sudden drop, discrete behave, and miss pointing can be seen in the measured pressure drop data and cannot be verified or estimated. To achieve a better result, the data in this case have been shifted.

\subsection{Data without outlier}

One of the cases observed in the collected data is the existence of outliers. To evaluate the effect of outliers, the measured data are filtered and outliers are eliminated from raw data, and again the pressure drop behavior is estimated. It was found that the final result and the coefficients of the clogging function $\left(\mathrm{K}_{1-3}, \mathrm{~L}_{1-3}\right)$ had no significant effect. Fig. 7 shows a comparison of filtered data and raw data. It should be noted that by removing the outliers here is similar to a low pass filter that filters high frequencies. The selected frequency should be less than the time intervals of data collection by the BAS, which is 15 minutes in the studied units. The added filtered data was provided to the authors about 50 days after the last study which can be seen in Fig. 7 . Moreover, as can be seen from this figure, there is an abnormal behavior in the pressure drop curve after day 
140. This behavior is exactly inversely related to the behavior of the temperature graph, which means that the pressure drop is only dependent on the inverse of the temperature.
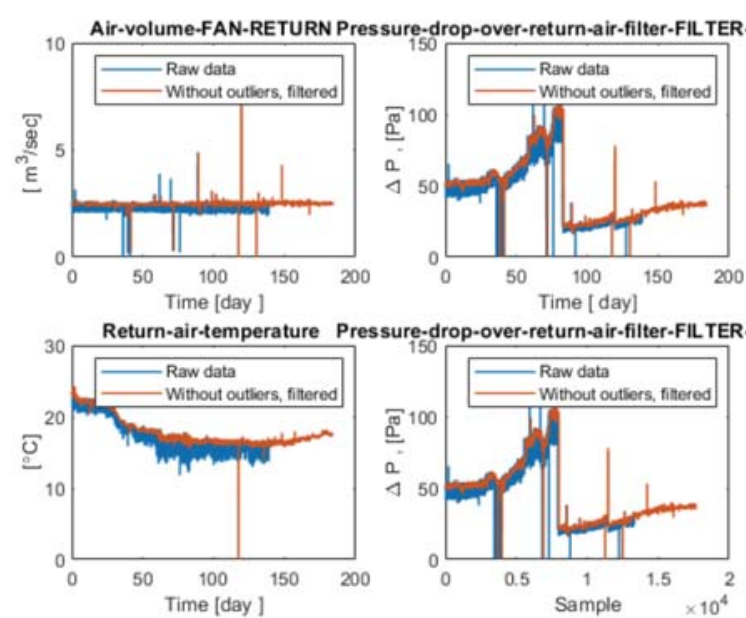

Fig. 7. Comparison of measured raw data and data without outliers

As shown in Fig. 8, this behavior occurs for all units, so a common factor causes this phenomenon.

$$
\Delta p(t)=\frac{f(t)_{\text {clog }} \dot{v}(t)}{T_{\text {inlet }}(t)}
$$

According to (7) and (10) consequently, it can be concluded that in this time period, the effect of clogging is as constant function and independent of time, $\mathrm{f}_{\text {clog }}(\mathrm{t})=$ cte, or in other words, AHU's filters are not exposed to any pollution. One reason for this behavior could be due to the corona epidemic period which leads to unused zones of AHUs.
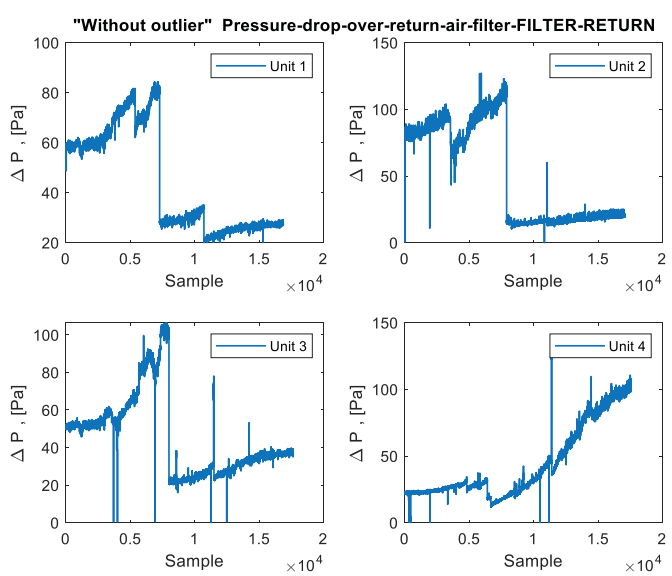

Fig. 8. Turning down behavior of pressure drop in all units

\section{Conclusion}

Based on the results presented above, the performance of the polynomial model for clogging filters leads to an acceptable correlation between the model and the test. However, in order to obtain a more accurate exponential clogging filter estimate, a larger amount of data (without any disruption) needs to be obtained within a year. This is because the clogging rate may be greater than that of a clean filter. In other words, the clogging decreases the ventilation through the filter exponentially, especially over a longer period of time.

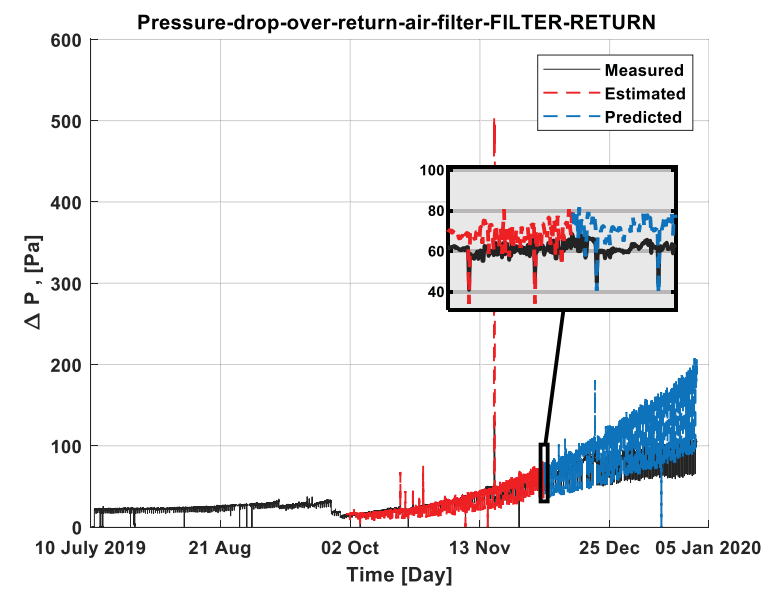

Fig. 9. Prediction result and tracking

Apart from simplicity, the method also covers all the modeling uncertainties observed in the articles. The accuracy of the method is proved by comparison with experimental data (Fig. 9).

It is worth mentioning that the influence of clogging on pressure drop can be expressed as a second-order polynomial function, which can be seen from the results due to the fact that the model and experimental data have acceptable traceability.

The results show that polynomial and exponential functions can be used to estimate filter clogging. However, the power function, $\mathrm{f}(\mathrm{t})_{\operatorname{clog} 3}=\mathrm{P}_{1} \mathrm{t}^{\mathrm{P}_{2}}+\mathrm{P}_{3}$ can also be examined and compared for future work.

\section{Acknowledgments}

This work has been partially conducted in the project "ICT programme" which was supported by the European Union through the European Social Fund. It was also supported by the Estonian Research Council grant PRG658.

\section{References}

R. M. Werner and L. A. Clarenburg, "Aerosol filters: Pressure Drop across Single-Component Glass Fiber Filters," Ind. Eng. Chem. Process Des. Dev., 1965.

G. W. Jackson and D. F. James, "The permeability of fibrous porous media," Can.J. Chem. Eng., 1986.

[3] S. A. Hosseini and H. V. Tafreshi, "Modeling particle filtration in disordered 2-D domains: A comparison with cell models," Sep. Purif. Technol., 2010.

[4] C. Kanaoka and S. Hiragi, "Pressure drop of air filter with dust load," J. Aerosol Sci., 1990.

[5] W. Bergman et al., " Enhanced filteration programm at LLL - a progress report.", 15th DOE Air Cleaning, 1978.

[6] P. Letourneau, P. Mulcey, and J. Vendel, “ Effect of dust loading on the pressure drop and efficiency of hepa filters.," Filtr. Sep., 1987.

[7] Y.Endo, D. R. Chen, and D. Y.H. Pui, "Effects 
of particle polydispersity and shape factor during dust cake loading on air filters," Powder Technol., 1998.

[8] D. Thomas, P. Contal, V. Renaudin, P. Penicot, D. Leclerc, and J. Vendel, "Modelling pressure drop in HEPA filters during dynamic filtration," J. Aerosol Sci., 1999.

[9] C. Ghiaus, A. Chicinas, and C. Inard, "Grey-box identification of air-handling unit elements," Control Eng. Pract., 2007.

[10] Y. Yao, K. Yang, M. Huang, and L. Wang, “A state-space model for dynamic response of indoor air temperature and humidity," Build. Environ., 2013.

[11] N. Hariharan and B. P. Rasmussen, "Parameter estimation for dynamic HVAC models with limited sensor information," in Proceedings of the 2010 American Control Conference, ACC 2010, 2010.

[12] Y. Yao, M. Huang, and J. Chen, "State-space model for dynamic behavior of vapor compression liquid chiller," Int. J. Refrig., 2013.

[13] M. Siemann, J. Kim, D. Oberholzer, and C. Sloop, "Performance of a residential building energy grey-box model using localized weather networks," ASHRAE Trans., 2013.

[14] J. E. Braun and N. Chaturvedi, "An inverse gray-box model for transient building load prediction," HVAC R Res., 2002.

[15] Y. Yao, M. Huang, J. Mo, and S. Dai, "Statespace model for transient behavior of water-toair surface heat exchanger," Int. J. Heat Mass Transf., 2013.

[16] S. Nyika, S. O. Holloway, W. T. Horton, and J. E. Braun, "Generalized performance maps for single- and dual-speed residential heat pumps," in ASHRAE Conference-Papers, 2014.

[17] R. L. Navale and R. M. Nelson, "Use of genetic algorithms and evolutionary strategies to develop an adaptive fuzzy logic controller for a cooling coil - Comparison of the AFLC with a standard PID controller," Energy Build., 2012.

[18] J. Wright and Y. Zhang, "Evolutionary synthesis of HVAC system configurations: Experimental results," HVAC R Res., 2008.

[19] Z. Ma and S. Wang, "Supervisory and optimal control of central chiller plants using simplified adaptive models and genetic algorithm," Appl. Energy, 2011.

[20] H. Moradi, F. Bakhtiari-Nejad, and M. SaffarAvval, "Multivariable robust control of an airhandling unit: A comparison between poleplacement and $\mathrm{H} \infty$ controllers," Energy Convers. Manag., 2012.

[21] X.Zhang, K. F. Fong, and S. Y. Yuen, “A novel artificial bee colony algorithm for HVAC optimization problems," HVAC R Res., 2013. 